\title{
Optimizing link atom parameters for DNA QM/MM simulations
}

\author{
Manuel Hitzenberger ${ }^{1} \cdot$ Manussada Ratanasak $^{2} \cdot$ Vudhichai Parasuk $^{2} \cdot$ \\ Thomas S. Hofer ${ }^{1}$
}

Received: 31 August 2015 / Accepted: 30 November 2015 / Published online: 5 February 2016

(C) The Author(s) 2016. This article is published with open access at Springerlink.com

\begin{abstract}
This work optimizes the link bond description of the quantum mechanical/molecular mechanical separation of deoxynucleosides. The nucleosides are separated at the $\mathrm{C}-\mathrm{N}$ bond between the nucleobase and the deoxyribose, with the former acting as the quantum mechanically described species. By using a flexible link atom-ansatz plus a harmonic potential to correct the energy deviation from a full quantum mechanical description, the potential energy well of the bond's stretching motion is mimicked with very high accuracy.
\end{abstract}

Keywords QM/MM · Biomolecules · DNA · Molecular dynamics $\cdot$ Link bond

\section{Introduction}

QM/MM-MD [1, 4, 5, 14, 15, 29, 37, 43, 44] simulations are of ever-growing interest for the investigation of metalion complexes [19, 28, 30, 38, 41] and biomolecules [36], such as proteins and ribonucleic acids, because of their methodological flexibility, the capacity to investigate subpicosecond events as well as the possibility to predict interor intramolecular interactions and even chemical reactions. Besides the higher accuracy, the main advantage of hybrid QM/MM-MD to classical MM-MD simulations that

Thomas S. Hofer

T.Hofer@uibk.ac.at

1 Theoretical Chemistry Division, Institute of General, Inorganic and Theoretical Chemistry, University of Innsbruck, Innrain 80 - 82, 6020 Innsbruck, Austria

2 Department of Chemistry, Faculty of Science, Chulalongkorn University, Phayathai Road, Patumwan, Bangkok 10330, Thailand utilize popular force fields like AMBER [9], CHARMM [8], OPLS [23] or GROMOS [35] is the ability to describe effects stemming from the intra- or intermolecular shifts of electron density for any desired chemical system. Due to this, phenomena such as charge transfers, the cleavage or formation of covalent bonds and polarization effects can be studied in-depth. Additionally, the influence of different spin states, Jahn Teller distortion and even electronic excitations can be investigated without having to resort to specifically tailored force fields.

One of the major challenges for that kind of investigation is a proper description of the interface between the QM and the MM region, especially if covalent bonds are reaching through the boundary between the two regions [27]. There exist a variety of approaches to describe that frontier bond, for example capping potentials [22, 26], generalized hybrid orbitals [16] or localized self-consisted field [3, 33, 40], but the simplest, yet very accurate [2] and therefore also highly popular $[27,36]$ method is hydrogen capping [39].

A big question regarding hydrogen-capping approaches is the proper placement of the inserted atom, so that its influence on the behavior of the surrounding atoms is minimized as much as possible. In a previous work [18], this was discussed in detail for amino acids, with the recommendation to use separate parameters for each type of sidechain to considerably improve the description of the link bond. In this work, the focus lies on the QM/MM separation of DNA nucleosides, with the $\mathrm{C}-\mathrm{N}$ bond between the deoxyribose and the nucleobase acting as the link bond. The investigated QM methods were RI-MP2 [31], B3LYP [6], B3LYP-D3 [17], BLYP [7] and BLYP-D3, as well as the resolution of identity (RI) [34] variants of the density functional theory $[20,25,32]$ (DFT) calculations. All 
computations were performed, utilizing a triple-zeta basis set $[12,45]$.

\section{Methodology}

The applied link atom scheme is entirely analog to the one used in the investigation of the amino acids [18]: the linkor capping atom $\left(L_{\mathrm{C}}\right)$ is positioned on the vector connecting the frontier $\mathrm{QM}$ atom $\left(L_{\mathrm{Q}}\right)$ and the frontier $\mathrm{MM}$ atom $\left(L_{\mathrm{M}}\right)$. Instead of a fixed distance for the $L_{\mathrm{Q}}-L_{\mathrm{C}}$ bond, a distance ratio $\rho$ is introduced, defining the position of $L_{\mathrm{C}}\left(\mathbf{r}_{L_{\mathrm{C}}}\right)$ as:

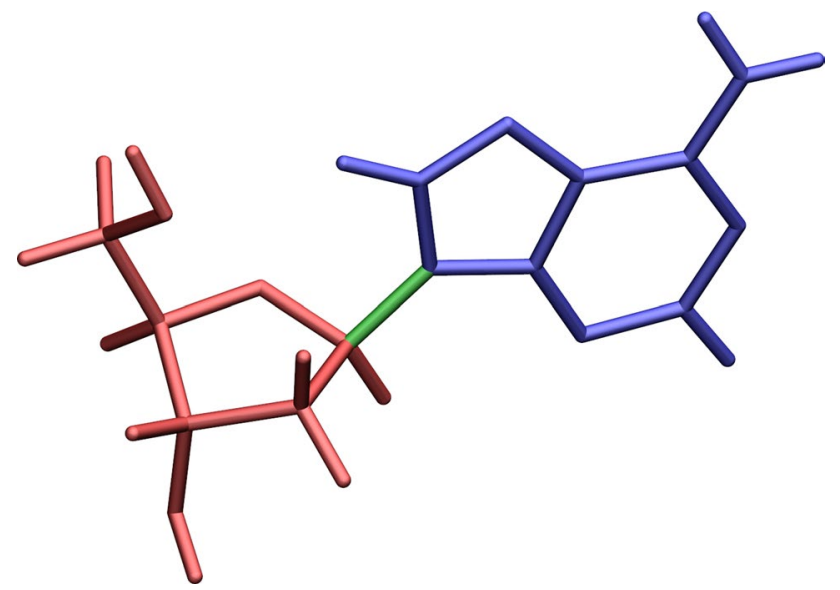

Fig. 1 Deoxyadenosine model system, used for the parametrization. The blue adenine base is described by one of the QM methods, while all atoms depicted in red are computed by the force field. Both regions are connected via the link bond (green)
$\mathbf{r}_{L_{\mathrm{C}}}=\rho \cdot\left(\mathbf{r}_{L_{\mathrm{M}}}-\mathbf{r}_{L_{\mathrm{Q}}}\right)+\mathbf{r}_{L_{\mathrm{Q}}}$

with $\mathbf{r}_{L_{\mathrm{Q}}}$ being the position of $L_{\mathrm{Q}}$ and $\mathbf{r}_{L_{\mathrm{M}}}-\mathbf{r}_{L_{\mathrm{Q}}}$ corresponding to the distance between the frontier atoms, respectively. To identify the best possible ratio (i.e., the one where the energy minima of the link bond and the full QM bond coincide), model calculations of the nucleosides deoxyadenosine (A), deoxyguanosin (G), deoxythymidine (T) and deoxycytidine $(\mathrm{C})$ have been conducted. All model systems were set up by initial full QM geometry optimization utilizing TURBOMOLE 6.5 [42]. Solvation effects were approximated implicitly using the COSMO [24] model. Atomic orbitals of $\mathrm{H}, \mathrm{C}, \mathrm{N}$ and $\mathrm{O}$ were described by the ccpVTZ $[12,45]$ triple-zeta basis set and the D3 method was applied, according to Grimme et al. [17].

In the QM/MM calculations, all nucleobases were computed via one of the QM methods, while deoxyribose was described by the AMBER-99SB [21] force field (see Fig. 1).

The system's total energy is evaluated for the minimum distance $r_{0}$ of the $L_{Q}-L_{\mathrm{M}}$ bond, obtained from the initial geometry optimization, as well as for a set of slightly higher and lower distances and several different values of $\rho$. The resulting energy-distance graphs are compared to that of a full QM description of the same system (see Fig. 2a). Once the ideal value for $\rho$ is identified, the zero points of both descriptions are aligned to one another by subtraction of the respective energy minimum $\left(E_{\mathrm{min}}\right)$. The remaining difference can now be obtained by subtracting the $\mathrm{QM} / \mathrm{MM}$ energy $\left(E_{\mathrm{QM} / \mathrm{MM}}\right)$ from the one of the full $\mathrm{QM}$ calculation $\left(E_{\mathrm{QM}}\right)$ and described by a harmonic potential of the form:

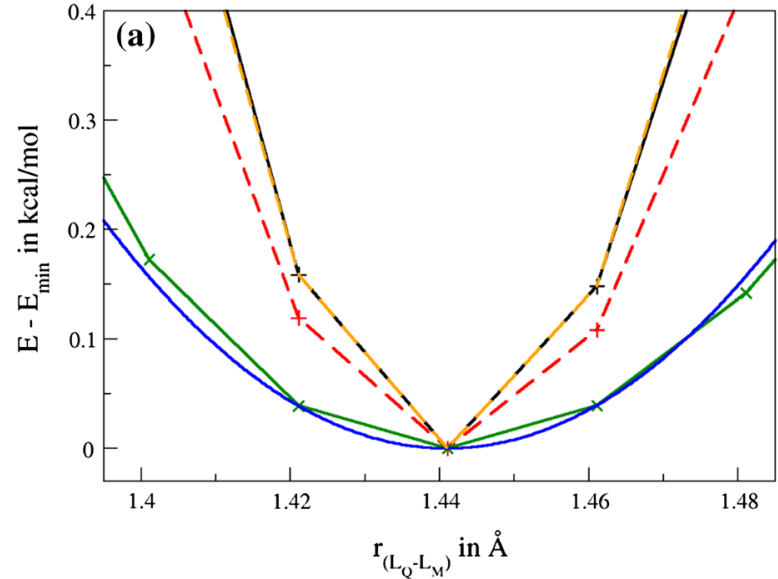

Fig. 2 a Graphical depiction of the parametrization process for the RI-MP2 representation of deoxyadenosine. The black line represents the potential energy well of the full QM description, whereas the red, dashed line stems from a QM/MM energy scan employing the ideal distance ratio of 0.6978 and the green line shows the energy difference between the two. If the harmonic potential (blue) that is fitted

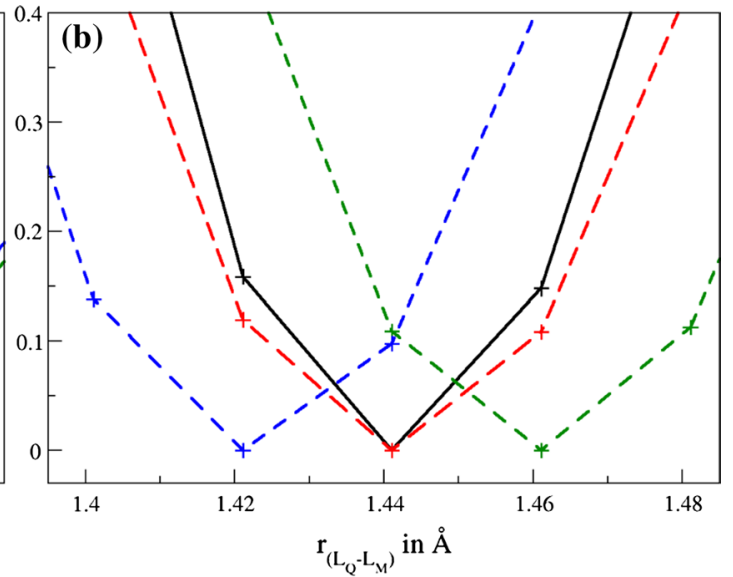

to the energy difference is added to the overall description of the link bond via a force field parameter, the resulting link bond description (orange, dashed line) exactly mimics the full QM case. b Comparison of a QM/MM scan, utilizing the ideal distance ratio (red, dashed) to scans with $\rho$, deviating by -0.01 (green, dashed) or +0.01 (blue, dashed). The full QM scan is again represented by a solid black line 


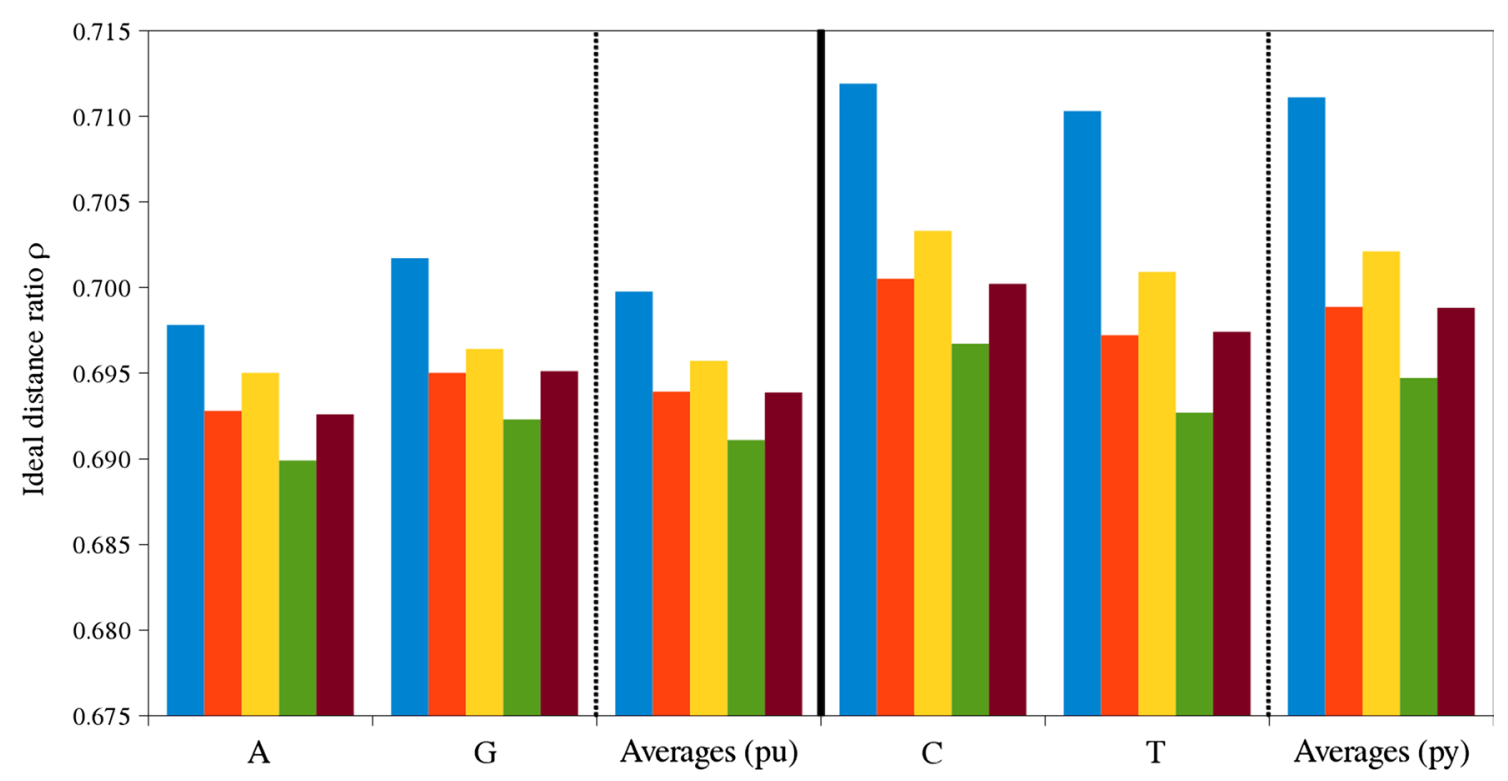

Fig. 3 Graphical representation of the obtained distance ratios for all investigated deoxynucleosides. The color coding of the data groups labeled A, G, C and T indicates the different QM methods, investigated in this work: blue RI-MP2, orange B3LYP, yellow B3LYP-D3,

Table 1 Overview over the obtained distance ratios $\rho$ for all investigated QM methods

\begin{tabular}{lllll}
\hline & $\mathrm{A}$ & $\mathrm{G}$ & $\mathrm{C}$ & $\mathrm{T}$ \\
\hline RI-MP2 & 0.6978 & 0.7017 & 0.7119 & 0.7103 \\
B3LYP & 0.6928 & 0.6950 & 0.7005 & 0.6972 \\
B3LYP-D3 & 0.6950 & 0.6964 & 0.7033 & 0.7009 \\
BLYP & 0.6899 & 0.6923 & 0.6967 & 0.6927 \\
BLYP-D3 & 0.6926 & 0.6951 & 0.7002 & 0.6974 \\
RI-BLYP & 0.6899 & 0.6923 & 0.6967 & 0.6925 \\
RI-BLYP-D3 & 0.6926 & 0.6951 & 0.7000 & 0.6971 \\
RI-B3LYP & 0.6928 & 0.6949 & 0.7003 & 0.6971 \\
RI-B3LYP-D3 & 0.6950 & 0.6964 & 0.7031 & 0.7008 \\
\hline
\end{tabular}

$V=\frac{k_{\mathrm{L}}}{2} \cdot\left(\left|\mathbf{r}_{L_{\mathrm{Q}}}-\mathbf{r}_{L_{\mathrm{M}}}\right|-r_{0}\right)^{2}$

with $\mathrm{V}$ representing the potential energy, $\mathbf{r}_{L_{\mathrm{Q}}}-\mathbf{r}_{L_{\mathrm{M}}}$ the distance between the two atoms that define the bond and $r_{0}, k_{L}$ the equilibrium distance and the force constant, respectively. $r_{0}$ and $k_{\mathrm{L}}$ can be obtained by fitting the harmonic potential to the energy difference, considering the three lowest points. The resulting harmonic potential can now be added to the overall QM/MM description of the link bond as a classical bond between $L_{\mathrm{Q}}$ and $L_{\mathrm{M}}$. Hence, the link bond is defined by a parameter set $\rho, r_{0}, k_{\mathrm{L}}$ and, provided proper parameters are used, accurately mimics the potential green BLYP and brown BLYP-D3. Also depicted are the recommended average values for pyrimidines (py) and purines (pu), respectively

energy well of a full QM description of the host bond—not only for the equilibrium position but also for configurations where the bond length is deviating from $r_{0}$.

As Fig. 2b shows, varying the distance ratio by a little margin, such as \pm 0.01 , results in bond lengths deviating by $\pm 0.02 \AA$ - which is the same error as one would introduce by switching from RI-MP2 to BLYP. The obtained graphs also suggest that at the full QM minimum, the potential well for $\rho=\rho$ (ideal $) \pm 0.01$ is still very much repulsive or attractive with a remaining potential energy of $\sim 0.1 \mathrm{kcal} /$ mol.

The description of the polar $\mathrm{C}-\mathrm{N}$ bond by $\mathrm{N}-\mathrm{H}$ also retains the direction of the resulting dipole, since the electronegativity of hydrogen is comparable to that of carbon and thus also lower than the electronegativity of nitrogen.

\section{Results and discussion}

The parametrization process has been performed following the scheme outlined in Ref. [18] and Sect. 2. The resulting distance ratios $\rho$ (depicted in Fig. 3; Table 1) indicate that there is (1) a large gap between the results obtained for MP2 and DFT and (2) a considerable difference between the pyrimidine based nucleosides and the purine ones. The data also suggests that the structural differences between the two pyrimidines, cytosine and thymine, are so small that the resulting ideal distance ratios are very similar to 
Table 2 Overview over the obtained parameters for the harmonic fit

\begin{tabular}{|c|c|c|c|c|c|c|c|c|}
\hline & \multicolumn{2}{|l|}{$\mathrm{A}$} & \multicolumn{2}{|l|}{$\mathrm{G}$} & \multicolumn{2}{|l|}{$\mathrm{C}$} & \multicolumn{2}{|l|}{$\mathrm{T}$} \\
\hline & $r_{0}$ & $k_{\mathrm{L}}$ & $r_{0}$ & $k_{\mathrm{L}}$ & $r_{0}$ & $k_{\mathrm{L}}$ & $r_{0}$ & $k_{\mathrm{L}}$ \\
\hline RI-MP2 & 1.441 & 196.11 & 1.447 & 179.90 & 1.456 & 140.14 & 1.456 & 133.35 \\
\hline B3LYP & 1.454 & 125.42 & 1.455 & 144.25 & 1.465 & 102.94 & 1.467 & 98.23 \\
\hline B3LYP-D3 & 1.454 & 140.02 & 1.449 & 153.39 & 1.465 & 98.69 & 1.466 & 93.97 \\
\hline BLYP & 1.467 & 79.94 & 1.468 & 97.53 & 1.475 & 52.80 & 1.483 & 24.69 \\
\hline BLYP-D3 & 1.465 & 97.56 & 1.465 & 101.74 & 1.479 & 55.79 & 1.482 & 37.38 \\
\hline RI-B3LYP & 1.455 & 125.58 & 1.455 & 148.46 & 1.465 & 95.19 & 1.468 & 79.18 \\
\hline RI-B3LYP-D3 & 1.453 & 150.88 & 1.447 & 157.26 & 1.464 & 96.73 & 1.467 & 97.79 \\
\hline RI-BLYP & 1.468 & 87.78 & 1.469 & 91.56 & 1.482 & 57.91 & 1.482 & 43.36 \\
\hline RI-BLYP-D3 & 1.465 & 95.57 & 1.464 & 102.83 & 1.477 & 61.56 & 1.482 & 61.60 \\
\hline
\end{tabular}

Values for $r_{0}$ are given in $\AA$ and $k_{\mathrm{L}}$ in $\mathrm{kcal} / \mathrm{mol} / \AA^{2}$

\begin{tabular}{|c|c|c|c|c|c|c|c|c|}
\hline & \multicolumn{2}{|l|}{ A } & \multicolumn{2}{|l|}{ G } & \multicolumn{2}{|l|}{$\mathrm{C}$} & \multicolumn{2}{|l|}{$\mathrm{T}$} \\
\hline & $r_{\mathrm{QM}}$ & $k_{\mathrm{QM}}$ & $r_{\mathrm{QM}}$ & $k_{\mathrm{QM}}$ & $r_{\mathrm{QM}}$ & $k_{\mathrm{QM}}$ & $r_{\mathrm{QM}}$ & $k_{\mathrm{QM}}$ \\
\hline RI-MP2 & 1.441 & 797.63 & 1.448 & 717.93 & 1.456 & 710.12 & 1.456 & 709.13 \\
\hline B3LYP & 1.455 & 649.03 & 1.455 & 653.33 & 1.467 & 629.99 & 1.468 & 635.29 \\
\hline B3LYP-D3 & 1.454 & 666.59 & 1.453 & 718.05 & 1.466 & 651.54 & 1.468 & 643.16 \\
\hline BLYP & 1.469 & 568.20 & 1.469 & 573.70 & 1.479 & 554.29 & 1.485 & 546.93 \\
\hline BLYP-D3 & 1.466 & 588.73 & 1.466 & 597.37 & 1.479 & 567.98 & 1.483 & 566.29 \\
\hline
\end{tabular}

Values for $r_{\mathrm{QM}}$ are given in $\AA$ and $k_{\mathrm{QM}}$ in $\mathrm{kcal} / \mathrm{mol} / \AA^{2}$
Table 3 Full QM force constants $k_{\mathrm{QM}}$ and $\mathrm{C}-\mathrm{N}$ distances $r_{\mathrm{QM}}$ $\mathrm{kcal} / \mathrm{mol} / \AA^{2}$ ). In order to assess whether the observed deviations of $k_{\mathrm{L}}$ stem from the chosen QM method or are an artifact of the QM/MM treatment, the full QM force constants $k_{\mathrm{QM}}$ were obtained by fitting a harmonic potential to the innermost three data points of the full QM scan. The results, shown in Table 3 indicate that (1) the strength of the force constants is indeed strongly dependent upon the chosen QM description (ranging from $\sim 560 \mathrm{kcal} / \mathrm{mol} / \AA^{2}$ for BLYP and $\sim 640 \mathrm{kcal} / \mathrm{mol} / \AA^{2}$ for B3LYP to $\sim 730 \mathrm{kcal} /$ $\mathrm{mol} / \AA^{2}$ for RI-MP2), thus implying that the deviation of $k_{\mathrm{L}}$ does not stem from the QM/MM treatment, and (2) the force constants $k_{\mathrm{QM}}$ of the DFT methods deviate between 547 (BLYP scan of deoxythymidine) and $718 \mathrm{kcal} / \mathrm{mol} / \AA^{2}$ (B3LYP-D3 scan of deoxyguanosine) and the average $k_{\mathrm{QM}}$ of all DFT scans is approximately $613 \mathrm{kcal} / \mathrm{mol} / \AA^{2}$. With a potential maximum error of the parametrization lying at $\sim 130 \mathrm{kcal} / \mathrm{mol} / \AA^{2}$ which is roughly $21 \%$ of the total force constant, it is clear that separate harmonic fit parameters for both, BLYP and B3LYP methods, as well as for the purine and pyrimidine bases are necessary. It is also obvious that RI-MP2 calculations demand different parameters altogether. However, the influence of the D3-correction to the energy correction terms is so small that individual parameters for D3 and non-D3 DFT methods do not seem necessary. The final suggested parameters are presented in Table 4. Two things are apparent when looking at the 
Table 4 Suggested QM/MM link bond parameters for purines and pyrimidines. $r_{0}$ are given in $\AA$ and $k_{\mathrm{L}}$ in $\mathrm{kcal} / \mathrm{mol} /$ $\AA^{2}$, respectively

\begin{tabular}{llllllll}
\hline & \multicolumn{1}{l}{ Purine } & \multicolumn{3}{l}{ Pyrimidine } \\
\cline { 2 - 3 } & $\rho$ & $r_{0}$ & $k_{\mathrm{L}}$ & & $\rho$ & $r_{0}$ & $k_{\mathrm{L}}$ \\
\hline RI-MP2 & 0.6998 & 1.456 & 136.748 & & 0.7103 & 1.450 & 162.375 \\
B3LYP & 0.6939 & 1.453 & 140.77 & & 0.6989 & 1.466 & 98.46 \\
B3LYP-D3 & 0.6957 & & & & 0.7021 & & \\
BLYP & 0.6911 & 1.466 & 94.19 & & 0.6947 & 1.480 & 42.66 \\
BLYP-D3 & 0.6939 & & & & 0.6988 & &
\end{tabular}

results: firstly, for the distance ratios the influence of the investigated nucleoside's nature becomes more prominent, the more accurate the chosen QM method is: the ideal distance ratios for BLYP (without dispersion correction) show the least deviation from one another (the difference between the mean values for purine and pyrimidine are just 0.0036). As soon as dispersion correction is employed, the variation of results is much larger (0.0049) and the distance ratios are almost identical to those of B3LYP (without dispersion correction). The MP2-based results show the highest sensitivity regarding the nature of the nucleobase with a purine-pyrimidine deviation of 0.0105 . Secondly, whereas dispersion correction has a very large influence on the values of $\rho$, this is not true for $r_{0}$ and $k_{\mathrm{L}}$ so that combined values for DFT and DFT-D3 can be safely used. Instead, an even stronger discrimination between the DFT methods has to be made. This is due to the fact that the resulting predicted bond lengths ( $\left.r_{\mathrm{QM}}\right)$ and force constants $\left(k_{\mathrm{QM}}\right)$ vary quite a bit, as given in Table 3. By calculating distance ratios, this differences are canceled out to a certain extend because they are directly related to the ideal $\mathrm{C}-\mathrm{N}$ distance, as well as the length of a $\mathrm{N}-\mathrm{H}$ bond, as predicted by the selected QM method (see Eq. 1). Hence, if BLYP overestimates the length of the $\mathrm{C}-\mathrm{N}$ bond compared with B3LYP, the $\mathrm{N}-\mathrm{H}$ distance is simultaneously overestimated by a similar scale. Therefore, the resulting distance ratios can be very similar nevertheless. This, however, is not true if one looks at the harmonic potential: since this represents the energy difference between the full QM and the QM/MM description of the bond stretching, it is directly dependent only of the predicted bond length and energy. In effect, the methodological characteristics are brought to bear to a much larger extend.

It should be noted that in the QM/MM framework, used for finding above parameters, classical angle and dihedral terms were included in the calculations if at least one MM atom was present in the definition. Often angle and dihedral terms with only QM atoms at their center are excluded in order to prevent double counting [13]. Inclusion or exclusion of said terms, however, has absolutely no influence on the obtained values for $\rho, k_{\mathrm{L}}, r_{0}$ as only the bond length between $L_{\mathrm{Q}}$ and $L_{\mathrm{M}}$ was varied, and therefore all contributions from angle and dihedral terms remained constant throughout. This would of course not hold true for simulations at a finite temperature. Another potential influence stems from the way $\mathrm{q}\left(L_{\mathrm{M}}\right)$, the charge of $L_{\mathrm{M}}$, was treated. In the used approach, the charge was neglected in the embedding scheme in order to prevent over-polarization. An alternative method would be the distribution of $\mathrm{q}\left(L_{\mathrm{M}}\right)$ to its remaining binding partners [10] (only for the embedding - the force field still uses the original set of charges). While a variation of charges can surely be expected to have an influence, the fact that for the investigated nucleosides the charges $\mathrm{q}\left(L_{\mathrm{M}}\right)$ provided by AMBER-99SB are all in the region between 0.01 and 0.07 , this influence is only very minor. It has been shown that even for molecules where $\mathrm{q}\left(L_{\mathrm{M}}\right)$ is considerably larger (between -0.13 and -0.26 ), the influence on the parameters is still very small [18]. A much larger [18] influence can be expected if the AMBER94 charges used in the majority of AMBER force field parametrizations are replaced by a different set (e.g., AMBER03-charges [11]).

\section{Conclusion}

The obtained results indicate that, depending on the level of theory being utilized in the description of the QM part of the simulation, a careful choice of the used parameters can improve the quality of the description considerably, without additional computational effort. The purine and the pyrimidine bases are chemically dissimilar enough to warrant independent distance ratios in all cases. However, it does not seem to be necessary to discriminate between adenine/guanine and cytosine/thymine, respectively. Generally, separate distance ratios $\rho$ for all investigated QM methods are suggested, however, due to the fact that those ratios are also very dependent of dispersion correction, sometimes differences between methods can be levelled out. As for the correction terms, which are far more sensitive to the nature of the chosen method than to dispersion correction, the method-specific influences are much more pronounced. Therefore, different $r_{0}$ and $k_{\mathrm{L}}$ parameters for RI-MP2, B3LYP and BLYP are necessary in order to exactly mimic the potential energy well of the full QM C-N bond by the utilized link atom approach. 
Acknowledgments Open access funding provided by University of Innsbruck and Medical University of Innsbruck. Financial support for this work by a Ph.D. scholarship of the Leopold-Franzens-University of Innsbruck for M. Hitzenberger is gratefully acknowledged. M. Ratanasak was financially supported by the "Erasmus Mundus Action 2 (EMA2) Project Swap and Transfer" program.

Open Access This article is distributed under the terms of the Creative Commons Attribution 4.0 International License (http://creativecommons.org/licenses/by/4.0/), which permits unrestricted use, distribution, and reproduction in any medium, provided you give appropriate credit to the original author(s) and the source, provide a link to the Creative Commons license, and indicate if changes were made.

\section{References}

1. Aaqvist J, Warshel A (1993) Simulation of enzyme reactions using valence bond force fields and other hybrid quantum/classical approaches. Chem Rev 93:2523

2. Amara P, Field MJ (2003) Evaluation of an ab initio quantum mechanical/molecular mechanical hybrid-potential link-atom method. Theor Chem Acc 109:43-52

3. Assfeld X, Rivail JL (1996) Quantum chemical computations on parts of large molecules: the ab initio local self consistent field method. J Comput Chem 263:100-106

4. Bakowies D, Thiel W (1996) Hybrid models for combined quantum mechanical and molecular mechanical approaches. J Phys Chem 100(25):10580-10594

5. Bash PA, Field MJ, Karplus M (1987) Free energy perturbation method for chemical reactions in the condensed phase: a dynamic approach based on a combined quantum and molecular mechanics potential. J Chem Am Soc 109(26):8092-8094

6. Becke A (1993) A new mixing of Hartree-Fock and local density functional theories. J Chem Phys 98:1372-1377

7. Becke AD (1988) Density-functional exchange-energy approximation with correct asymptotic behavior. Phys Rev A 38:3098

8. Brooks BR, Bruccoleri RE, Olafson BD, States DJ, Swaminathan S, Karplus M (1983) CHARMM: a program for macromolecular energy, minimization, and dynamics calculations. J Comput Chem 4:187-217

9. Case DA, III TEC, Darden T, Gohlke R Jr, Luo R KMM Jr, Onufriev A, Simmerling C, Wang B, Woods R (2005) The amber biomolecular simulation programs. J Comput Chem 26:1668-1688

10. de Vries A, Sherwood P, Collins S, Simon J, Rigby A, Anthony M, Rigutto M, Kramer GJ (1999) Zeolite structure and reactivity by combined quantum-chemical-classical calculations. J Phys Chem B 103:6133-6141

11. Duan Y, Wu C, Chowdhury S, Lee MC, Xiong G, Zhang W, Yang R, Cieplak P, Luo R, Lee T, Caldwell J, Wang J, Kollman P (2003) A point-charge force field for molecular mechanics simulations of proteins based on condensed-phase quantum mechanical calculations. J Comput Chem 24(16):1999-2012

12. Dunning TH (1989) Gaussian basis sets for use in correlated molecular calculations. i. the atoms boron through neon and hydrogen. J Chem Phys 90:1007

13. Eurenius KO, Chatfield DC, Brooks BR, Hodoscek M (1996) Enzyme mechanisms with hybrid quantum and molecular mechanical potentials. I. Theoretical considerations. Int J Quant Chem 60:1189-1200

14. Gao J (1993) Potential of mean force for the isomerization of DMF in aqueous solution: a Monte Carlo QM/MM simulation study. JACS 115:2930
15. Gao J (1996) Hybrid quantum and molecular mechanical simulations: an alternative avenue to solvent effects in organic chemistry. Acc Chem Res 29:298

16. Gao J, Amara P, Alhambra C, Field MJ (1998) A generalized hybrid orbital (GHO) method for the treatment of boundary atoms in combined QM/MM calculations. J Phys Chem A 102(24):4714-4721

17. Grimme S, Antony J, Ehrlich S, Krieg H (2010) A consistent and accurate ab initio parametrization of density functional dispersion correction (DFT-D) for the 94 elements H-Pu. J Chem Phys 132: 154,104

18. Hitzenberger M, Hofer TS (2015) Probing the range of applicability of structure- and energy-adjusted $\mathrm{qm} / \mathrm{mm}$ link bonds. J Comput Chem 39(26):1929-1939

19. Hofer TS, Weiss AKH, Randolf BR, Rode BM (2011) Hydration of highly charged ions. Chem Phys Lett 512(4-6):139-145

20. Hohenberg P, Kohn W (1964) Inhomogeneous electron gas. Phys Rev B 13:4272-4298

21. Hornak V, Abel R, Okur A, Strockbine B, Roitberg A, Simmerling C (2006) Comparison of multiple amber force fields and development of improved protein backbone parameters. Proteins Struct Funct Bioinform 65:712-725

22. Ihrig AC, Schiffmann C, Sebastiani D (2011) Specific quantum mechanical/molecular mechanical capping-potentials for biomolecular functional groups. J Chem Phys 135:214,107

23. Jorgensen WL, Maxwell DS, Tirado-Rives J (1996) Development and testing of the OPLS all-atom force field on conformational energetics and properties of organic liquids. J Chem Theor Comput 118(45):11,236-22,336

24. Klamt A, Schüürmann G (1993) Cosmo: a new approach to dielectric screening in solvents with explicit expressions for the screening energy and its gradient. J Chem Soc Perkin Trans 2(5):799-805

25. Kohn W, Sham LJ (1965) Self-consistent equations including exchange and correlation effects. Phys Rev A 140:1133-1138

26. Komin S, Sebastiani D (2008) Optimization of capping potentials for spectroscopic parameters in hybrid quantum mechani$\mathrm{cal} /$ mechanical modeling calculations. J Chem Theor Comput 5:1490-1498

27. Lin H, Truhlar DG (2007) QM/MM: what have we learned, where are we, and where do we go from here. Theor Chem Acc 117:185-199

28. Lutz OMD, Hofer TS, Randolf BR, Rode BM (2012) Computational study of the cerium(iii) ion in aqueous environment. Chem Phys Lett 539-540:50-53

29. Lyne PD, Hodoscek M, Karplus M (1990) A hybrid QM-MM potential employing Hartree-Fock or density functional methods in the quantum region. J Phys Chem A 103:3462

30. Moin T, Hofer TS, Pribil AB, Rode BM (2010) A quantum mechanical charge field molecular dynamics study of fe $2+$ and fe3+ ions in aqueous solutions. Inorg Chem 49(11):5101-5106

31. Møller C, Plesset M (1934) Note on an approximation treatment for many-electron systems. Phys Rev 46(7):618-622

32. Parr RG (1983) Ann Rev Phys Chem 34:631-656

33. Philipp DM, Friesner RA (1999) Mixed ab initio qm/mm modeling using frozen orbitals and tests with alanine dipeptide and tetrapeptide. J Comput Chem 20(14):1468-1494

34. Ren X, Rinke P, Blum V, Wieferink J, Tkatchenko A, Sanfilippo A, Reuter K, Scheffler M (2012) Resolution-of-identity approach to Hartree-Fock, hybrid density functionals, RPA, MP2 and GW with numeric atom-centered orbital basis functions. N J Phys 14(5):053,020

35. Scott WRP, Hünenberger PH, Tironi IG, Mark AE, Billeter SR, Fennen J, Torda AE, Huber T, Krüger P, van Gunsteren WF (1999) The GROMOS biomolecular simulation program package. J Phys Chem A 103:3596-3607 
36. Senn HM, Thiel W (2007) QM/MM studies of enzymes. Curr Opin Chem Biol 11(2):182-187

37. Senn HM, Thiel W (2009) QM/MM methods for biomolecular systems. Angew Chem Int Ed 48:1198-1229

38. Sikander AS, Hofer TS, Bhattacharjee A, Lim L, Pribil AB, Randolf BR, Rode BM (2009) Beryllium(ii): the strongest structureforming ion in water? A QMCF MD simulation study. J Phys Chem B 113(27):9289-9295

39. Singh UC, Kollman PA (1986) A combined ab initio quantum mechanical and molecular mechanical method for carrying out simulations on complex molecular systems: applications to the $\mathrm{Ch}_{3} \mathrm{Cl}+\mathrm{Cl}^{-}$exchange reaction and gas phase protonation of polyethers. J Comput Chem 7:7180

40. Théryt V, Rinaldi D, Rivail J-L, Maigret B, Ferenczy G (1994) Quantum mechanical computations on very large molecular systems: the local self-consistent field method. J Comput Chem 15(3):269-282
41. Tirler AO, Hofer TS (2014) Structure and dynamics of the uranyl tricarbonate complex in aqueous solution: Insights from quantum mechanical charge field molecular dynamics. J Phys Chem B 11pm8:12938-12951

42. Turbomole V6.5 (2013) A development of University of Karlsruhe and Forschungszentrum Karlsruhe GmbH, 19892007, TURBOMOLE GmbH, since 2007. http://www.turbomole.com

43. Warshel A (2002) Molecular dynamics simulations of biological reactions. Acc Chem Res 35:385

44. Warshel A, Levitt M (1976) Theoretical studies of enzymic reactions: dielectric, electrostatic and steric stabilization of the carbenium ion in the reaction of lysozyme. J Mol Biol 103:227

45. Woon DE, Dunning TH (1993) Gaussian basis sets for use in correlated molecular calculations. iii. the atoms aluminum through argon. J Chem Phys 98:1358 